\title{
Aspectos higiênico-sanitários no processo produtivo dos alimentos em escolas públicas do Estado de Goiás, Brasil ${ }^{1}$
}

\author{
Sanitary aspects of food preparation \\ in public schools of Goiás, Brazil
}

Nair Augusta de Araújo Almeida GOMES²

Maria Raquel Hidalgo CAMPOS 3

Estelamaris Tronco MONEGO3

\section{R E S U M O}

\section{Objetivo}

Este estudo pretendeu monitorar as condições higiênico-sanitárias em cantinas de escolas públicas do estado de Goiás, investigadas na pesquisa Avaliação e Monitoramento da Qualidade dos Alimentos Oferecidos na Merenda Escolar, Goiás, desenvolvida de 2004 a 2007.

\section{Métodos}

A partir de 2004, foi realizada pesquisa em escolas públicas de Goiás envolvendo três etapas. Na primeira, de 2004 a 2005, foram diagnosticadas as condições higiênico-sanitárias das cantinas dessas escolas, utilizando-se check list. Na segunda etapa, de 2005 a 2007, promoveram-se ações de intervenção nas escolas com capacitação de pessoal técnico. Na terceira etapa, em 2010, foram monitoradas condições higiênico-sanitárias das cantinas em dezoito escolas participantes da primeira etapa, utilizando-se o mesmo check list a fim de comparar a dimensão higiênico-sanitária da alimentação escolar nos dois momentos. O projeto foi aprovado pelo Comitê de Ética em Pesquisa da Universidade Federal de Goiás.

\section{Resultados}

As escolas estudadas cumpriram parcialmente as exigências legais, visto que as inadequações dos itens avaliados - higiene pessoal, condições da edificação, equipamentos e utensílios, higiene operacional e processamento - estavam presentes em 44,9\% das cantinas investigadas no período de 2004 a 2005, e em 37,1\% das avaliadas na investigação atual, o que coloca em risco a qualidade higiênico-sanitária da alimentação produzida.

\footnotetext{
1 Artigo elaborado a partir da dissertação de NAAA GOMES, intitulada "Qualidade higiênico-sanitária da alimentação oferecida em escolas públicas do estado de Goiás”. Universidade Federal de Goiás; 2011.

2 Secretaria Municipal de Educação de Goiânia, Departamento de Alimentação Educacional. R. 227, 564, Setor Leste Universitário, 74605-080, Goiânia, GO, Brasil. Correspondência para/Correspondence to: NAAA GOMES. E-mail: <nairaugustaalmeida@yahoo.com.br>.

3 Universidade Federal de Goiás, Faculdade de Nutrição. Goiânia, GO, Brasil.
} 


\section{Conclusão}

Comparando as etapas de investigação, verificou-se melhora de conformidade apenas nos itens do bloco higiene operacional. Tais resultados reforçam a necessidade de adequação física das cantinas escolares e de capacitação periódica, com vistas à melhoria permanente na produção da alimentação escolar.

Termos de indexação: Alimentação escolar. Boas práticas de manipulação. Segurança alimentar e nutricional.

\section{A B S T R A C T}

\section{Objective}

This study aimed to monitor the cleanness of public school canteens of Goiás state, investigated during the study "Avaliação e Monitoramento da Qualidade dos Alimentos Oferecidos na Merenda Escolar" (Assessment and Monitoring of School Food Quality), Goiás, done from 2004 to 2007.

\section{Methods}

Starting in 2004, a three-stage survey was done in public schools of Goiás. During the first phase, from 2004 to 2005, a checklist was used to assess the cleanness of schools canteens. In the second phase, from 2005 to 2007, interventions were done at the schools to train the relevant personnel. The third phase, done in 2010, consisted of monitoring the 18 school canteens that participated in the first phase using the same checklist to compare the sanitary quality of their meals in the two periods. The study was approved by the Universidade Federal de Goiás Research Ethics Committee.

\section{Results}

The study schools complied only in part with the legislation, as the items personal hygiene, facility conditions, equipment and utensils, operational hygiene and processing were inadequate in $44.9 \%$ of the school food services investigated from 2004 to 2005 and in $37.1 \%$ of the ones investigated during the present study, jeopardizing the sanitary quality of the preparations.

\section{Conclusion}

Comparison of the two phases of this investigation showed that only operational hygiene improved. These results reinforce the need of improving the pertinent school facilities and providing periodical training to those involved in food preparation to improve the sanitary quality of school foods.

Indexing terms: School feeding. Good manipulation practices. Food security.

\section{N T R O D U Ç Ã O}

O Programa Nacional de Alimentação Escolar (PNAE) destaca-se, entre os programas sociais do Brasil, pelo tempo de existência e pela dimensão do atendimento prestado, haja vista ter atendido no ano de 2010 a 45,6 milhões de estudantes matriculados na educação básica, de crianças, de jovens e de adultos ${ }^{1,2}$. O PNAE é considerado uma estratégia de promoção da Segurança Alimentar e Nutricional $(\mathrm{SAN})^{(4,5)}$ por garantir a oferta de alimentação nos intervalos das atividades escolares a todos os alunos de escolas públicas e filantrópicas do País ${ }^{3}$.

As refeições produzidas nas Unidades de Alimentação e Nutrição (UAN) escolares devem atender às necessidades nutricionais dos alunos, oferecendo-Ihes produtos adequados sob os aspectos sensorial e nutricional, mas, sobretudo, produtos seguros quanto à condição higiênico-sanitária para a proteção e promoção da saúde dos beneficiários ${ }^{5}$. A qualidade higiênico-sanitária

\footnotetext{
4 O conceito de Segurança Alimentar e Nutricional, segundo o Consea ${ }^{4}$, consiste na realização do direito de todos ao acesso regular e permanente a alimentos de qualidade, em quantidade suficiente, sem comprometer o acesso a outras necessidades essenciais, tendo como base práticas alimentares promotoras da saúde que respeitem a diversidade cultural e que seja ambiental, cultural, econômica e socialmente sustentáveis.

5 Alimento Seguro, segundo a ISO 2005 (International Standard- Food Safety Management Systems-Requirements for Any Organization in the Food Chain. ISO/FDIS 22000,2005), é aquele que não causará dano ao consumidor quando preparado e/ou consumido de acordo com seu uso pretendido.
} 
como fator de segurança alimentar tem sido muito estudada e discutida, uma vez que a incidência de Doenças Transmitidas por Alimentos (DTA) vem aumentando em nível mundial ${ }^{6}$.

No Brasil, do total de surtos de DTA notificados no período de 1999 a 2008, 10,7\% ocorreram em instituições de ensino ${ }^{7}$. Dentre as possíveis causas dessas enfermidades, encontram-se as práticas inadequadas de higiene e o processamento de alimentos por pessoas inabilitadas ${ }^{8}$. Os cuidados higiênicos no processo produtivo e a educação dos manipuladores envolvidos na preparação, processamento e serviços são limites cruciais para a prevenção da maioria das DTA 9 .

A produção de alimentos seguros é prática necessária, em especial nas escolas públicas, espaço que atende uma clientela vulnerável quanto aos aspectos nutricional e socioeconômico, e para a qual, por vezes, esse alimento constitui a única refeição do dia ${ }^{10}$. Portanto, a ocorrência de um surto de DTA nesse ambiente tende a configurar-se como um problema de saúde pública.

Dessa forma, é essencial o conhecimento acerca das Boas Práticas de Fabricação (BPF) na produção da alimentação escolar. Uma vez estabelecidos os pontos críticos no que diz respeito aos aspectos higiênico-sanitários desse processo, tais informações poderão contribuir para promover as políticas públicas no sentido de estabelecer melhorias na execução do PNAE a fim de garantir a produção de refeições adequadas e saudáveis e proteger a saúde dos alunos.

O estudo de que trata o presente artigo pretendeu monitorar, em 2010, as condições higiênico-sanitárias das cantinas de escolas públicas do estado de Goiás, previamente investigadas na pesquisa interinstitucional - Avaliação e Monitoramento da Qualidade dos Alimentos Oferecidos na Merenda Escolar, Goiás, desenvolvida de 2004 a 2007. Foi possível comparar, portanto, a dimensão higiênico-sanitária da alimentação escolar nesses dois momentos.

\section{MÉ T O D O S}

O estudo caracterizou-se como transversal ${ }^{11}$ e foi realizado em UAN de escolas públicas do estado de Goiás no período de maio a junho de 2010. A partir do ano de 2004, foi realizada uma pesquisa interinstitucional em escolas públicas do estado de Goiás, intitulada Avaliação e Monitoramento da Qualidade dos Alimentos Oferecidos na Merenda Escolar, Goiás, que envolveu a Faculdade de Nutrição (FANUT) da Universidade Federal de Goiás (UFG), a Superintendência de Vigilância Sanitária e Ambiental da Secretaria de Estado da Saúde (VISA-GO/SES) e o Ministério Público do Estado de Goiás.

Tal investigação constituiu-se de três etapas. Na primeira, de 2004 a 2005, foram diagnosticadas as condições higiênico-sanitárias das cantinas dessas escolas por meio de um check list de boas práticas de manipulação de alimentos, elaborado a partir das exigências da legislação sanitária vigente. Na segunda etapa, de 2005 a 2007, foram promovidas ações de intervenção nas escolas com programa de capacitação de pessoal técnico (nutricionistas e manipuladores) e produção de material instrucional. Na terceira etapa, em 2010, objeto deste estudo, foram monitoradas as condições higiênico-sanitárias das cantinas em dezoito escolas participantes da primeira etapa, utilizando-se o mesmo check list, a fim de comparar a dimensão higiênico-sanitária da alimentação escolar nos dois momentos. O projeto foi aprovado pelo Comitê de Ética em Pesquisa/UFG, protocolo $n^{\circ}$ 019/06.

Para definição da amostra, considerou-se a divisão estabelecida pela Secretaria de Estado da Saúde de Goiás dos municípios em cinco macrorregionais que se subdividem em quinze microrregionais, das quais faz parte a microrregional de Goiânia. Para aplicação do instrumento diagnóstico, das 3527 escolas existentes no ano de 2004, foram sorteadas aleatoriamente $20 \%$, o que totalizou 704 escolas.

Foram considerados, para a terceira etapa, os municípios da microrregional de Goiânia, 
e, destes, as escolas que participaram da fase de diagnóstico e de intervenção (etapas 1 e 2). Foram incluídas escolas do município de Goiânia, e, em virtude da capacidade operacional do pesquisador, escolas de municípios situados em cada uma das principais vias de acesso à capital e distando até $50 \mathrm{~km}$. Assim, a amostra foi composta por 14 municípios e 18 unidades escolares, sendo 15 localizadas no perímetro urbano e três na zona rural dos respectivos municípios.

Durante a coleta de dados, foram realizadas inspeções nas UAN escolares por meio de check list sobre BPF, elaborado com base na Resolução RDC n² 216/2004, da Agência Nacional de Vigilância Sanitária (Anvisa) do Ministério da Saúde ${ }^{12}$, aplicado pessoalmente pela pesquisadora, devidamente treinada, que também participou da coleta no período de 2004 a 2005 (etapa 1).

Para o preenchimento do instrumento, utilizou-se o método da observação visual e as informações fornecidas pelos diretores das escolas. Ao todo, o check list foi composto por 49 itens de verificação, agrupados em cinco blocos: bloco 1) higiene pessoal, avaliada por nove questões; bloco 2) condições da edificação (higiene e conservação ambiental), avaliadas por dezenove questões; bloco 3) equipamentos e utensílios, avaliados por cinco questões; bloco 4) higiene operacional, avaliada por onze questões; bloco 5) processamento, avaliado por cinco questões. Os itens avaliados foram classificados em "conformes" e "não conformes", de acordo com as recomendações da legislação sanitária de alimentos ${ }^{12}$.
Para classificação do grau de conformidade das UAN em relação ao check list, consideraram-se: grupo 1 - satisfatório, com 76\% a 100\% dos critérios atendidos; grupo 2 - regular, atendendo de 51 a $75 \%$ dos critérios e o grupo 3 - insatisfatório, com 0 a $50 \%$ dos critérios atendidos ${ }^{13}$.

Os resultados encontrados foram comparados aos obtidos no diagnóstico das UAN escolares, realizado no período de 2004 a 2005.

Na análise das variáveis, utilizou-se o programa Statistical Package for the Social Science (SPSS) (versão 17.0). Foi utilizado o Teste McNemar para comparar os resultados obtidos na verificação das BPF no período de diagnóstico com a avaliação atual. Para verificar associação entre a participação dos manipuladores em capacitações com as demais variáveis das BPF, utilizou-se o Teste Exato de Fisher. Foi empregado o Teste dos Sinais para comparar a classificação qualitativa do nível de conformidade das UAN. Foram feitas análises de associação entre as variáveis independentes por meio do teste $t$ de Student para comparação de médias. O nível de significância considerado foi de $5 \%$.

\section{RES U LT A D OS}

Observando-se os resultados por bloco de avaliação do check list e comparando-se os dois estudos, de modo geral, não houve melhora no percentual de conformidade dos quesitos avaliados, com diferença estatística significante apenas no bloco higiene operacional (Tabela 1).

Tabela 1. Percentual de conformidade por bloco de avaliação do check list, de acordo com a Resolução RDC n 216/ANVISAMMS, Brasil, na etapa de diagnóstico da pesquisa, realizada de 2004 a 2005, e na etapa de monitoramento, realizada em 2010, em escolas públicas do estado de Goiás, Brasil.

\begin{tabular}{|c|c|c|c|c|c|c|c|}
\hline \multirow{2}{*}{ Identificação do bloco } & \multirow{2}{*}{$\mathrm{n}$} & \multicolumn{2}{|c|}{$2004-2005$} & \multicolumn{2}{|c|}{2010} & \multirow{2}{*}{$t$} & \multirow{2}{*}{ Valor $p$} \\
\hline & & Média & Desvio-Padrão & Média & Desvio-Padrão & & \\
\hline Higiene pessoal & 18 & 43,82 & 22,38 & 51,85 & 16,17 & $-1,265$ & 0,223 \\
\hline Condições da edificação & 18 & 47,37 & 15,64 & 52,63 & 19,27 & $-1,057$ & 0,305 \\
\hline Equipamentos e utensílios & 18 & 63,33 & 25,90 & 64,44 & 25,26 & $-0,117$ & 0,908 \\
\hline Higiene operacional & 18 & 58,59 & 22,10 & 80,31 & 9,98 & $-3,634$ & 0,002 \\
\hline Processamento & 18 & 62,22 & 26,47 & 64,44 & 24,31 & $-0,325$ & 0,749 \\
\hline
\end{tabular}

Teste $t$ pareado. M: Média; DP: Desvio-Padrão. 
Da etapa de diagnóstico para a fase de monitoramento, verificaram-se, quanto ao nível de conformidade, a redução de sete para duas UAN escolares classificadas como grupo 3 (insatisfatório) e o aumento de UAN classificadas como grupo 2 (regular), ou seja, uma variação de $50 \%$ para $78 \%$, sem diferença estatisticamente significante $(p=0,344)$. Em ambos os momentos de investigação, apenas duas (11\%) das UAN avaliadas encontraram-se no grupo 1 (satisfatório).

Considerando-se os itens avaliados no bloco higiene pessoal - participação em treinamento e ausência de afecções e infecções -, os resultados apontaram melhora quando comparados aos dados da etapa de diagnóstico $(p<0,05)$. No entanto, ao considerarem-se os hábitos higiênicos adequados, os resultados pioraram na etapa de monitoramento $(p<0,05)$ (Figura 1).

Quanto ao bloco condições da edificação, considerando as duas investigações, foram estatisticamente significantes as diferenças quanto às condições de higiene e à execução de limpeza periódica dos reservatórios de água $(p=0,008)$ e tratamento e destino adequado dos outros resíduos (sólidos e gasosos) produzidos $(p=0,002)$ (Figura 2).

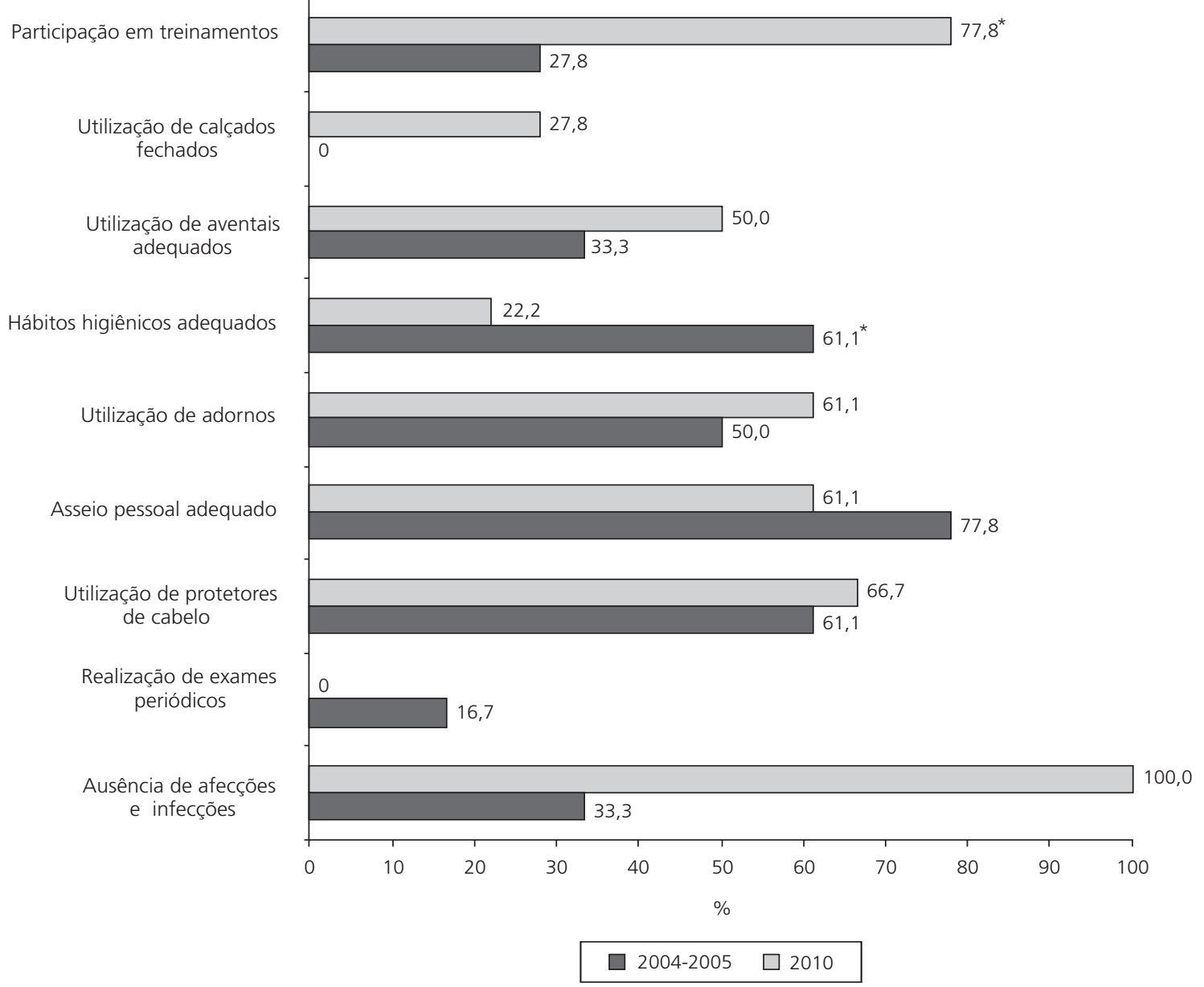

Figura 1. Porcentagem de adequação dos itens do bloco higiene pessoal, avaliados por check list, elaborado de acordo com a Resolução RDC n: 216 ANVISA/MS, na etapa de diagnóstico, realizada de 2004 a 2005 e na etapa de monitoramento, realizada em 2010, em escolas públicas $(n=18)$ do estado de Goiás, Brasil.

Nota: *Diferença estatisticamente significante ( $p<0,05$ - Teste de McNemar). 


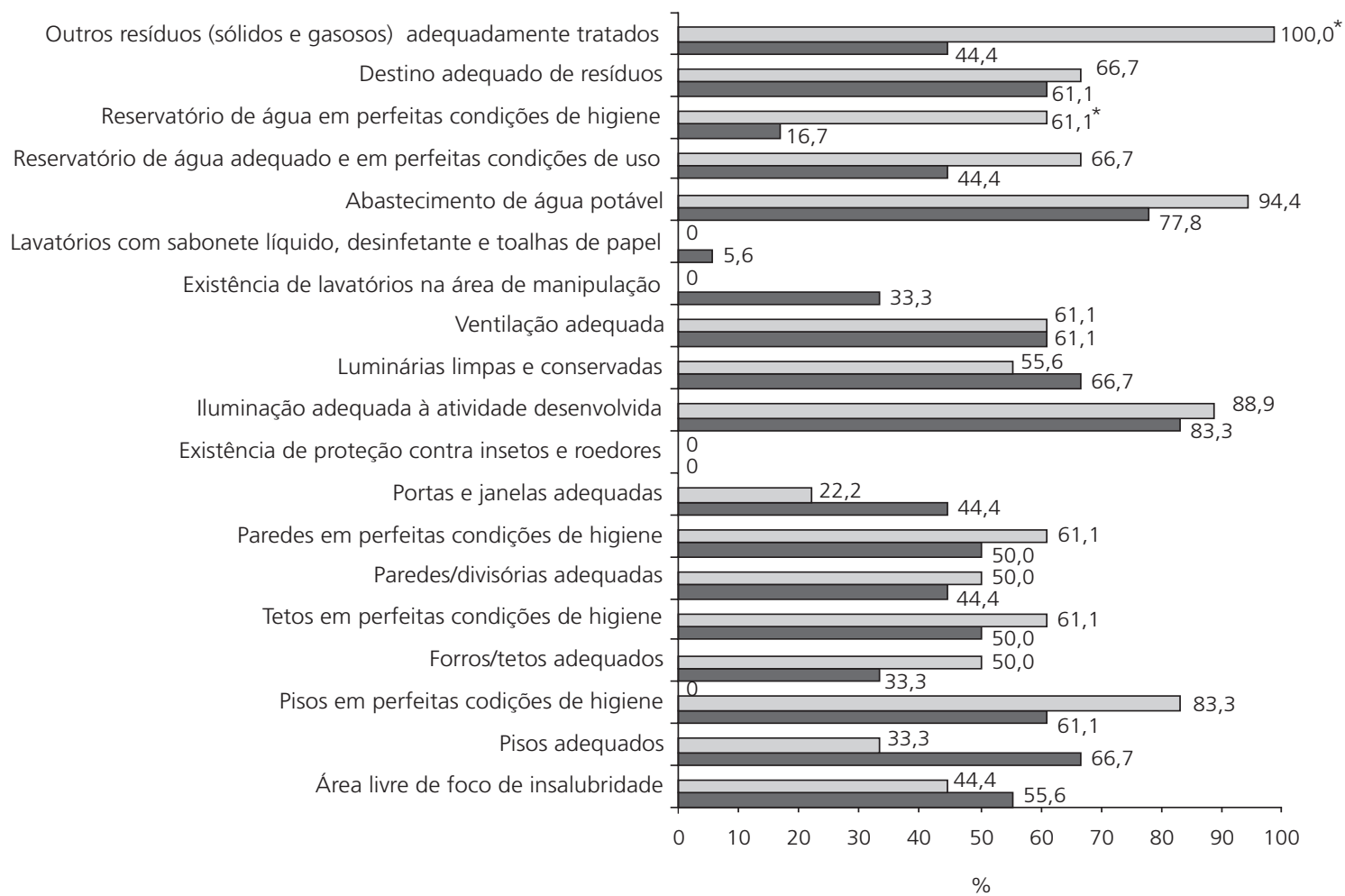

$2004-2005 \square 2010$

Figura 2. Porcentagem de adequação dos itens do bloco condiçães da edificação, avaliados por check list, elaborado de acordo com a Resolução RDC n. 216 ANVISA/MS, na etapa de diagnóstico, realizada de 2004 a 2005, e na etapa de monitoramento, realizada em 2010, em escolas públicas $(n=18)$ do estado de Goiás, Brasil.

Nota: *Diferença estatisticamente significante ( $p<0,05$ - Teste de McNemar).

No bloco higiene operacional, foram considerados estatisticamente significativos o registro dos alimentos nos órgãos de fiscalização competentes $(p=0,008)$, a existência de controle na utilização das matérias-primas $(p=0,001)$ e a utilização de carne e leite de procedência conhecida $(p=0,008)$ (Figura 3), verificando-se melhora no percentual de conformidade na etapa de monitoramento.

Não foram encontrados resultados estatísticos significativos quando comparados os itens do bloco equipamentos e utensílios (Figura 4). Verificou-se que embora haja melhorado o percentual de adequação em $82 \%$ dos itens avaliados sobre condições de processamento no estudo atual, a significância estatística foi encontrada apenas quanto ao conhecimento e à aplicação dos critérios de tempo e temperatura para descon- gelamento e manipulação dos alimentos que requerem temperaturas controladas $(p=0,021)$.

$\mathrm{Na}$ associação entre participação dos manipuladores em capacitação e itens dos blocos higiene pessoal, condições da edificação, equipamentos e utensílios, higiene operacional e processamento, percebeu-se que, na etapa de diagnóstico, os itens controle de pragas e vetores urbanos $(p=0,044)$ e critério de obediência de tempo e temperatura para o armazenamento de alimentos prontos $(p=0,047)$ foram considerados estatisticamente significantes. Em 13 das 18 UAN avaliadas, os manipuladores relataram não ter participado de capacitações; em 12 destas (92,3\%), não era adotado o procedimento de controle integrado de pragas e vetores urbanos, e em 10 (76,9\%), não se obedecia ao critério de tempo e temperatura. Dos cinco locais em que os manipu- 


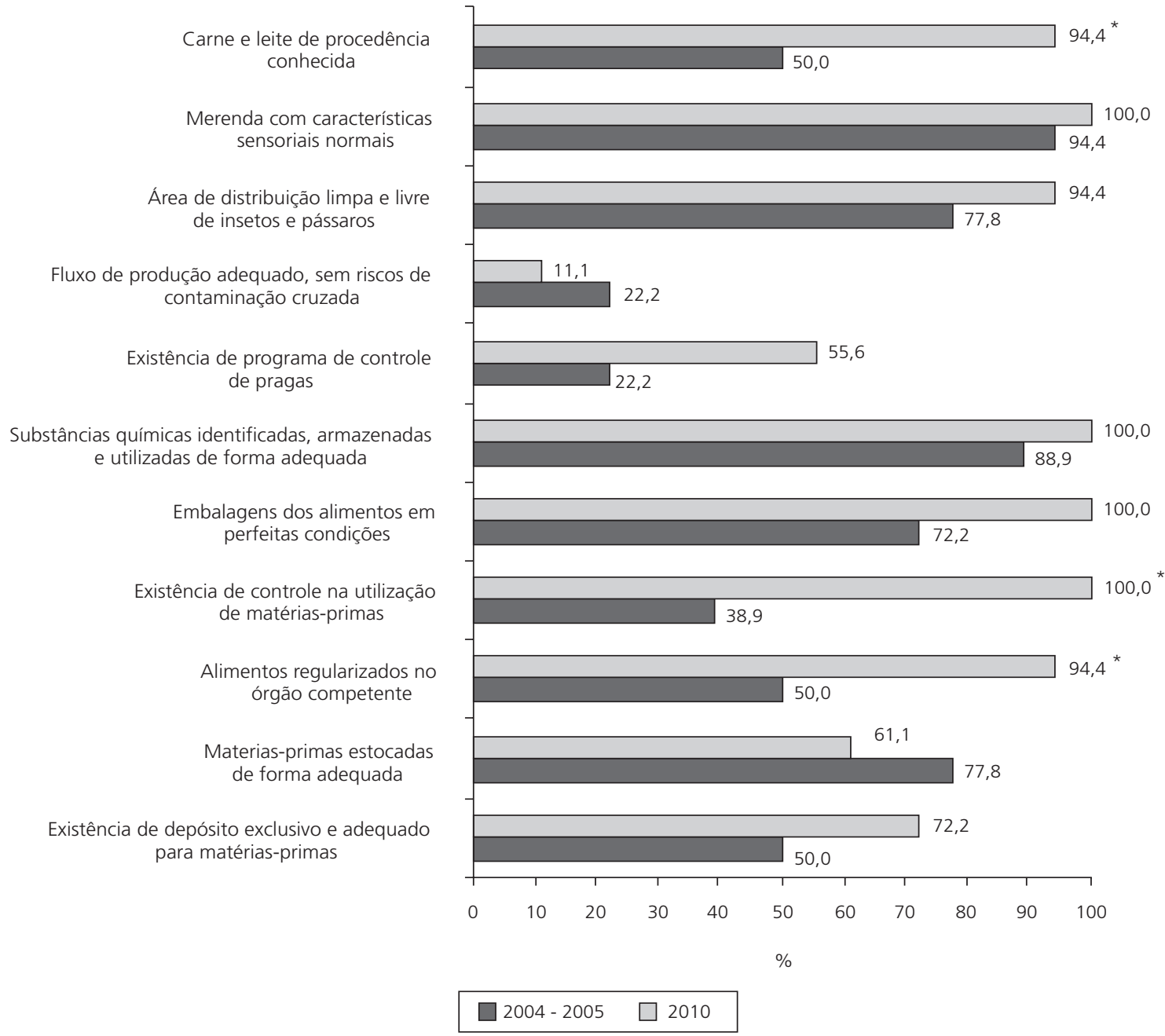

Figura 3. Percentual de conformidade dos itens do bloco higiene operacional, avaliados por check list elaborado de acordo com a Resolução RDC n. 216 ANVISA/MS, na etapa de diagnóstico, realizado de 2004 a 2005 e na etapa de monitoramento, realizada em 2010, em escolas públicas ( $n=18$ ) do estado de Goiás, Brasil.

Nota: *Diferença estatisticamente significante ( $p<0,05$ - Teste de McNemar).

ladores relataram ter participado de capacitações, três $(60,0 \%)$ adotavam o procedimento de controle das pragas e quatro $(80,0 \%)$ obedeciam ao critério de tempo e temperatura.

\section{I S C U S S Ã O}

Tanto na etapa de diagnóstico (2004-2005) quanto na etapa de monitoramento (2010) foram identificadas não conformidades em todas as UAN escolares visitadas, o que sugere risco de contaminação na produção de alimentos nesses locais.
O que se observou na classificação das UAN da etapa de diagnóstico para a etapa de monitoramento confirma que houve poucos avanços nos procedimentos de boas práticas dessas unidades. Essa constatação reforça a necessidade de um envolvimento maior dos gestores, do Conselho de Alimentação Escolar, de nutricionistas e manipuladores para assegurar as condições humanas e físico-estruturais necessárias à produção de refeições seguras.

Outros estudos classificaram o grau de conformidade das UAN em relação ao check list. 
Obediência aos critérios de tempo e temperatura para o armazenamento de alimentos prontos

\section{Reaproveitamento de alimentos \\ Alimentos servidos imediatamente após o preparo \\ Obediência aos critérios de higienização de frutas, verduras e folhosos \\ Aplicação dos critérios de tempo e temperatura para descongelamento e manipulação \\ Armazenamento de utensílios e equipamentos em local apropriado \\ Bancadas e mesas em material, conservação e limpeza aquados \\ Utensílios em material, conservação e limpeza adequados \\ Equipamentos adequados para conservação sob refrigeração}

Equipamentos em superfície, conservação, limpeza e funcionamento adequados
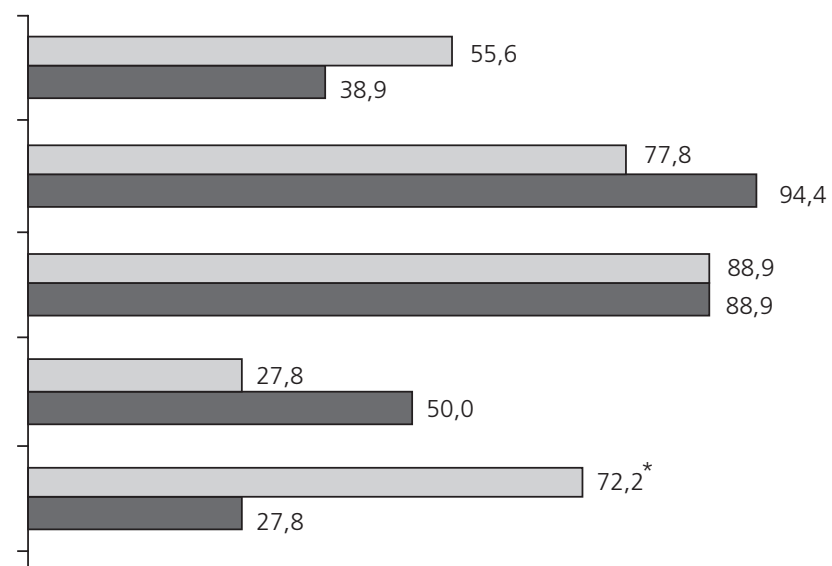

61,1 50,0

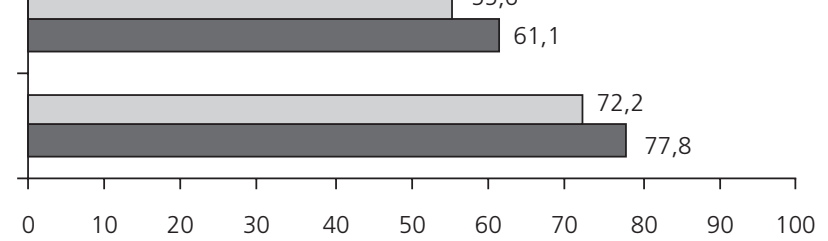

$\%$

Figura 4. Percentual de conformidade dos itens dos blocos equipamentos e utensilios e processamento, avaliados por check list elaborado de acordo com a Resolução RDC n²16 ANVISAMMS, na etapa de diagnóstico 2004 a 2005 e na etapa de monitoramento, realizada em 2010, em escolas públicas $(n=18)$ do estado de Goiás, Brasil.

Nota: *Diferença estatisticamente significante ( $p<0,05$ - Teste de McNemar).

Pesquisa realizada por Cardoso et al. ${ }^{14}$ em escolas públicas da cidade de Salvador (BA) obteve resultados diferentes dos encontrados no presente estudo: $0,4 \%$ das unidades escolares foram categorizadas no grupo $1 ; 42,6 \%$, no grupo 2 ; e $57,0 \%$, no grupo 3. Machado et al. ${ }^{15}$, que pesquisaram UAN de Organizações Não Governamentais (ONG) do município de Toledo (PR), verificaram que $10,0 \%$ classificavam-se no grupo 1 ; $50,0 \%$ no grupo 2 e $40,0 \%$ no grupo 3 .

Cardoso et al. ${ }^{14}$ consideram fundamental que as escolas possam proporcionar melhores condições de higiene pessoal aos manipuladores a partir de instalações adequadas aos serviços, de modo a viabilizar a obtenção de alimentos mais seguros. Kochanski et al. ${ }^{16}$ acrescentam que investimentos em estrutura adequada para o funcionamento e adoção de medidas de higienização podem reverter em maior segurança microbiológica nos estabelecimentos de alimentação coletiva.

O maior percentual de não conformidade foi observado no bloco higiene pessoal. Dentro desse bloco, o quesito hábitos higiênicos adequados buscou avaliar a lavagem cuidadosa das mãos e os atos físicos passíveis de contaminar o alimento: espirrar, tossir, fumar ou manipular dinheiro ${ }^{12}$. Oliveira et al. ${ }^{8}$, em pesquisa em creches públicas do município de São Paulo, e 
Cardoso et al. ${ }^{14}$, que pesquisaram escolas públicas da cidade de Salvador (BA), observaram que, respectivamente, $81,2 \%$ e $66,4 \%$ dos manipuladores de alimentos conversavam, assobiavam ou cantavam durante o preparo dos alimentos; em $6,2 \%$ e $51,7 \%$ dessas unidades verificou-se que esses profissionais não lavavam as mãos antes de manipular os alimentos e de tocar em qualquer outro material. Tais resultados são preocupantes uma vez que o comportamento de risco do manipulador compromete a segurança dos alimentos produzidos $^{14}$.

É sobejamente reconhecida na literatura a importância das práticas higiênicas adequadas pelos manipuladores como forma de garantir a sanidade dos alimentos e, assim, reduzir a incidência de DTA ${ }^{17}$. A legislação preconiza que manipuladores com lesões e ou sintomas de enfermidades que possam comprometer a qualidade higiênico-sanitária dos alimentos - condições avaliadas no quesito ausência de afecções e infecções - devem ser afastados da atividade de preparação de alimentos enquanto persistirem essas condições de saúde ${ }^{12}$. Essa mesma normativa acrescenta que esses profissionais devem ser supervisionados e capacitados periodicamente ${ }^{12}$. Nos estabelecimentos avaliados durante a etapa de diagnóstico, a normativa não estava sendo cumprida, o que colocava em risco a integridade do manipulador, a inocuidade do alimento produzido e, por conseguinte, a saúde dos comensais. Os resultados encontrados foram semelhantes aos de estudo conduzido em creches públicas e filantrópicas de São Paulo e em escolas públicas de Salvador (BA), nas quais foram evidenciadas deficiência em relação à lavagem cuidadosa das mãos antes de manipular o alimento e inadequações durante a manipulação8,14.

Quanto às condições da edificação, o percentual de conformidade observado na etapa de monitoramento em relação à etapa de diagnóstico foi superior em 11 dos 19 itens avaliados. Essa condição é condizente com relatos de diretores sobre reparos e pequenas reformas promovidas em algumas escolas após a realização da etapa de diagnóstico, entretanto o padrão disposto na legislação ${ }^{12}$ ainda não foi alcançado.

O nível de conformidade verificado aponta a necessidade de maior investimento financeiro nas edificações. As UAN observadas apresentavam porte de cozinha doméstica, corroborando a conclusão de Teo et al. ${ }^{18}$ de que elas foram incorporadas à arquitetura escolar como uma improvisação tanto em termos de espaço físico quanto de quantidade e de capacidade de equipamentos disponíveis, condição que pode limitar a composição dos cardápios e oferecer riscos de contaminação das refeições produzidas.

Cardoso et al. ${ }^{14}$, quando da aplicação de check list referente ao planejamento físico e funcional das cantinas escolares, observaram 58,3\% de condições insatisfatórias e $41,7 \%$ de regulares. Tal constatação reforça a necessidade da presença do nutricionista na equipe de técnicos responsáveis pelo planejamento da estrutura física de UAN escolar, pois esse profissional é habilitado, por formação, para planejar, gerenciar e avaliar as UAN ${ }^{19}$ : sua presença se faz importante na elaboração da planta física a fim de propor adequações à legislação sanitária vigente.

Na etapa atual da investigação, constatou-se percentual de adequação superior ao da etapa de diagnóstico nos itens destino adequado de outros resíduos produzidos e limpeza e higienização do reservatório de água. A legislação exige que o reservatório de água esteja livre de rachaduras, vazamentos, infiltrações e descascamentos, entre outros defeitos, em adequado estado de higiene e conservação, bem como com um intervalo máximo de higienização de seis meses, comprovado mediante registros da operação ${ }^{12}$.

De acordo com Esperança \& Marchioni ${ }^{20}$, a preocupação com as questões ambientais, a exemplo do destino apropriado para o lixo, deve-se traduzir em ações programáticas e contínuas. Cardoso et al. ${ }^{14}$ consideram o manejo de resíduos um preocupante fator, que, além de comprometer a higiene ambiental, expõe os escolares a situações de risco uma vez que aumenta as chances de proliferação de pragas e vetores nas UAN esco- 
lares. Os resíduos produzidos devem ser coletados e estocados em local fechado e isolado da área de preparação e armazenamento dos alimentos de forma a evitar focos de contaminação e atração de vetores e pragas urbanas ${ }^{12}$.

Estudo realizado em 105 Unidades Produtoras de Refeições (UPR) comercias de Florianópolis (SC) identificou como alimentos seguros produtos de qualidade adquiridos de bons fornecedores, respeitando o prazo de validade, armazenados adequadamente e manipulados seguindo as normas de higiene e da vigilância sanitária ${ }^{21}$. De acordo com Cardoso et al. ${ }^{14}$, na cadeia produtiva de alimentos, a obtenção da matéria-prima de boa qualidade, com a procedência garantida pelos órgãos de inspeção, é considerada requisito para a garantia da qualidade e da inocuidade do produto final, e está relacionada à saúde dos comensais uma vez que alimentos de procedência indeterminada não passam por inspeção sanitária ou registro em órgãos públicos, procedimento que visa ao controle dos produtos e têm o intuito de garantir que o alimento esteja próprio para o consumo.

Ao pesquisar os equipamentos e os utensílios, na etapa atual de estudo, foram observados talheres, pratos e canecas de polipropileno com necessidade de reposição. Observaram-se, ainda, condições inadequadas de uso de móveis e equipamentos para conservação de alimentos sob temperatura controlada.

Oliveira et al. ${ }^{8}$ observaram, nas cantinas de creches públicas, $80 \%$ dos equipamentos e utensílios fora dos padrões higiênico-sanitários. Segundo os autores, o estado de conservação e o funcionamento dos equipamentos e utensílios, bem como suas condições de limpeza, influenciam na qualidade final do alimento produzido.

Os utensílios disponibilizados pela entidade executora do PNAE nos municípios são de material plástico (polipropileno), pouco comum no uso cotidiano dos alunos. Com o uso contínuo, eles podem sofrer alterações de coloração e de textura ${ }^{18}$, dificultando sua higienização e favorecendo o acúmulo de resíduos que podem levar à contaminação cruzada na alimentação escolar ${ }^{8}$. Segundo a Resolução RDC n² 216/2004, equipamentos e utensílios utilizados em UAN devem ser de material impermeável, estar em bom estado de conservação e passar por processos de manutenção adequados ${ }^{12}$.

A legislação sanitária recomenda orientações quanto ao procedimento de descongelamento de forma a evitar que as áreas superficiais dos alimentos se mantenham em condições favoráveis à multiplicação microbiana ${ }^{12}$. De modo contrário ao disposto, na etapa de monitoramento da pesquisa, em $27,8 \%$ das UAN visitadas, o descongelamento de carnes ocorreu à temperatura ambiente. Procedimento similar foi observado por Cardoso et al. ${ }^{22}$ em cantinas da Universidade Federal da Bahia, que apresentavam assim condições propícias à multiplicação de microrganismos. Figueiredo ${ }^{23}$ acrescenta que, após o descongelamento, a taxa de multiplicação de microrganismos é semelhante à do alimento fresco, podendo chegar a níveis elevados a ponto de desencadear eventos de DTA.

Os resultados encontrados na etapa de monitoramento retrataram que poucos itens avaliados apresentaram melhora depois das ações de capacitação, o que mostra que elas não resultaram em mudanças comportamentais. É possível que os treinamentos tradicionais sejam inadequados, pois desconsideram o conhecimento prévio e a realidade de vida dos manipuladores. Entretanto, há de se considerar que embora haja falhas no processo de capacitação, as concepções das boas práticas de alguns manipuladores são resultados desse processo. Considerando a associação observada entre a participação em capacitações e os demais itens avaliados, o controle integrado de pragas e vetores urbanos é de competência da gerência do serviço, cabendo ao manipulador informar sobre as exigências legais de execução do procedimento. A obediência de tempo e de temperatura para o armazenamento de alimentos prontos faz parte das boas práticas que o manipulador deve adotar na etapa de processamento. 
Leite et al. ${ }^{24}$ salientam a necessidade de programas de formação para os manipuladores de alimentos a fim de estabelecer e confirmar técnicas higiênico-sanitárias consistentes, já que são profissionais da educação e contribuem no ambiente escolar para o processo de aprendizagem dos alunos, o que tem repercussão direta na sustentação do direito à alimentação saudável e segura. Kraemer \& Aguiar ${ }^{25}$ consideram que os investimentos em treinamentos são pontuais e não têm como objetivo o desenvolvimento de competências, restringindo-se ao cumprimento da legislação sanitária vigente.

Gonzalez et al. ${ }^{26}$ questionam a eficácia dos treinamentos realizados não somente pelas metodologias empregadas, mas também pela frequência de sua aplicação, considerada insuficiente. Alguns autores reconhecem a necessidade de abordagens inovadoras nas capacitações em boas práticas, estruturadas a partir de práticas que preparam o manipulador para o trabalho, devendo considerar o ponto de vista, as características específicas do ambiente ocupacional e as influências socioculturais que incidem sobre a segurança alimentar27,28.

A construção de um modelo de formação que atenda as demandas específicas apresentadas pelos próprios participantes pode permitir uma ressignificação para práticas tradicionais de educação e proporcionar maior chance de êxito ${ }^{24}$, uma vez que recursos metodológicos utilizados nas atividades formativas, quando bem aceitos, podem interferir no nível de satisfação dos participantes ${ }^{29} \mathrm{e}$ favorecer a apropriação das novas informações.

De acordo com Bas et al. ${ }^{30}$, todos os manipuladores devem participar de atividades de capacitação quando admitidos na UAN, e, a partir de então, continuamente. Essa rotina possivelmente melhore a segurança dos alimentos na medida em que o conhecimento adquirido contribui para o aprimoramento das habilidades e motiva mudanças de atitude e comportamento no local de trabalho ${ }^{29}$. Vale ainda considerar a necessidade de trabalhar outros campos das habilidades profissionais, isto é, além do saber, o saber fazer, o saber ser e o saber conviver, o que poderá contribuir para a (re)construção das práticas e, consequentemente, das relações profissionais, e possibilitará mudanças nos processos de trabalho ${ }^{31}$.

Segundo Clayton et al. ${ }^{27}$, 85\% dos manipuladores de restaurantes de pequeno e médio porte do País de Gales consideram como obstáculos à efetivação das ações de segurança alimentar e nutricional o tempo reduzido, a estrutura física e o fluxo de produção inadequados, a falta de recursos humanos e materiais e o reconhecimento dos problemas pela gestão.

\section{CONCLUSÃO}

Comparadas as duas etapas de investigação, conclui-se que houve melhora no nível de conformidade apenas nos itens do bloco higiene operacional, o que não elimina o risco de contaminação na produção de alimentos nas UAN visitadas. As principais inadequações observadas em ambas etapas de estudo referem-se à deficiência de recursos humanos adequadamente qualificados e à ausência de supervisão continuada, bem como instalações físicas incompatíveis com a função a que se propõem.

O item higiene pessoal foi o que apresentou menor nível de conformidade, o que sugere que as capacitações em BPF não foram suficientes para sedimentar um conhecimento capaz de influenciar a prática correta do manipulador. São recomendados estudos que relacionem atividades formativas e mudanças de atitude e comportamento no local de trabalho.

A estrutura físico-funcional inadequada observada nas UAN provavelmente contribua para o nível de inconformidade encontrado no bloco higiene pessoal. Observou-se, em todas as escolas visitadas, a inexistência de lavatório exclusivo para higienização das mãos na área de manipulação, de sabonete líquido, de solução desinfetante e de toalhas de papel descartáveis. Tal constatação sugere a necessidade de um envolvimento maior dos gestores quanto à adequação das UAN escolares ao disposto na legislação sanitária vigente. 
Conclui-se, em suma, que as UAN das escolas investigadas cumpriram apenas parcialmente as exigências legais, uma vez que o nível de não conformidade encontrado configura maior porcentagem de itens classificados como regular quanto aos critérios exigidos, não garantindo, assim, a segurança desejada para a qualidade da alimentação escolar, mesmo considerando que a etapa de diagnóstico da pesquisa tenha ocorrido no ano de 2004, quando da promulgação da Resolução RDC n²16/2004 - normativa que estabelece os procedimentos para o alcance de padrões de qualidade que confiram segurança aos serviços de alimentação, incluindo as UAN escolares.

As realidades vivenciadas nas escolas corroboram o reconhecimento da necessidade de uma apropriação do PNAE por todos os atores envolvidos para que o programa atinja o propósito que o fundamenta: atender o direito do aluno de alimentar-se dignamente no ambiente escolar. Com vistas a alcançar esse propósito, sugerem-se algumas iniciativas, como uma legislação específica para funcionamento das UAN escolares; a contratação de nutricionistas para supervisão e orientação periódica das atividades desenvolvidas nas UAN; a regulamentação da ocupação de manipulador de alimentos e a realização de um trabalho de educação continuada para esse público.

Para a realização de novos estudos acerca da qualidade da alimentação escolar como forma de orientar a implantação das BPF, faz-se necessário o fortalecimento da parceria entre a academia e os órgãos públicos tanto de gestão escolar como de fiscalização sanitária, visando à melhoria de serviços para a comunidade e ao desenvolvimento de investigações de campo.

\section{A GRADECIMENTOS}

Os autores agradecem os secretários de Educação, diretores e manipuladores que viabilizaram a realização da pesquisa. Agradecimento especial à professora Márcia Armentano Clarck Reis.

\section{COLABORADORES}

NAAA GOMES colaborou na concepção e desenho do estudo, coleta, análise e interpretação dos dados, pesquisa bibliográfica e redação do artigo. MRH CAMPOS colaborou na concepção e desenho do estudo, redação e revisão crítica do artigo. ET MONEGO no desenho do estudo e revisão crítica do artigo.

\section{REFERÊ NCIAS}

1. Chaves LG, Mendes PNR, Brito RR, Botelho RBA. O programa nacional de alimentação escolar como promotor de hábitos alimentares regionais. Rev Nutr. 2009; 22(6):857-66. doi:10.1590/\$1415-52 732009000600007

2. Brasil. Ministério da Educação. Fundo Nacional de Desenvolvimento da Educação [Internet]. Alimentação escolar. [acesso 2012 mar 10]. Disponível em: <http://www.fnde.gov.br/index.php/aeapresentacao>.

3. Brasil. Agência Nacional de Vigilância Sanitária [Internet]. Resolução RDC $n^{\circ} 038$, de 16 de julho de 2009. [acesso 2010 nov 20]. Disponível em: <http:// www.fnde.gov.br/index.php/ae-legislacao>.

4. Conselho Nacional de Segurança Alimentar e Nutricional. III Conferência Nacional de Segurança Alimentar e Nutricional: por um desenvolvimento sustentável com soberania e segurança alimentar e nutricional. Relatório final. Brasília: CONSEA; 2007.

5. Cardoso RCV, Almeida RCC, Guimarães AG, Góes JAW, Santana AAC, Silva SA, et al. Avaliação da qualidade microbiológica de alimentos prontos para consumo servidos em escolas atendidas pelo Programa Nacional de Alimentação Escolar. Rev Inst Adolfo Lutz. 2010; 69(2): 208-13.

6. Akutsu RC, Botelho RA, Camargo EB, Sávio KEO, Araújo WC. Adequação das boas práticas de fabricação em serviços de alimentação. Rev Nutr. 2005; 18(3):419-27. doi: 10.1590/\$1415-5273200500 0300013

7. Brasil. Ministério da Saúde. Sistema de Vigilância em Saúde [Internet]. Análise epidemiologica dos surtos de doenças transmitidas por alimentos no Brasil. [acesso 2012 mar 5]. Disponível em: <http:// portal.saude.gov.br/portal/arquivos/pdf/surtos dta_15.pdf>.

8. Oliveira MN, Brasil ALD, Taddei, JAAC. Avaliação das condições higiênico-sanitárias das cozinhas de creches públicas e filantrópicas. Ciênc Saúde Coletiva. 2008; 13(3):1051-60. doi: 10.1590/S1413-81 232008000300028. 
9. Aguiar OB, Kraemer FB. Educação formal, informal e não-formal na qualificação profissional dos trabalhadores de alimentação coletiva. Nutrire Rev Soc Bras Aliment Nutr. 2010; 35(3):87-96.

10. Brasil. Ministério da Educação. Secretaria de Educação Básica [Internet]. Políticas de Alimentação Escolar [acesso 2012 mar 05]. Disponível em: <http:// portal.mec.gov.br/seb/arquivos/pdf/profunc/ 12_pol_aliment_escol.pdf>.

11. Medronho RA, Bloch KV, Luiz RR, Werneck GL. Epidemiologia. 2a ed. São Paulo: Atheneu; 2009.

12. Brasil. Agência Nacional de Vigilância Sanitária. Resolução RDC $n^{\circ} 216$, de 15 de setembro de 2004. [acesso 2010 nov 20]. Disponível em: <http:// www.biblioteca.sebrae.com.br/bds/BDS.nsf/ CF4EFE7D0F91614B832576250049D87C/\$File/ NT00041F3E.pdf>.

13. Brasil. Agência Nacional de Vigilância Sanitária [Internet]. Resolução RDC n²75, de 21 de outubro de 2002. [acesso 2010 nov 20]. Disponível em: <http://www.anvisa.gov.br/legis/resol/2002/275_ 02 rdc.htm>.

14. Cardoso RCV, Góes JAW, Almeida RCC, Guimarães AG, Barreto DL, Silva SA, et al.Programa nacional de alimentação escolar: há segurança na produção de alimentos em escolas de Salvador (Bahia)? Rev Nutr. 2010; 23(5):801-11. doi: 10.1590/S1415-527 32010000500010.

15. Machado AD, Strapazon MA, Massing LT, Moreira DG, Possamai GA, Gabriel CM, et al. Condições higiênico-sanitárias nos serviços de alimentação de organizações não governamentais de Toledo/PR. Nutrire: 2009; 34(3):141-51.

16. Kochansky S, Pierozan MK, Mossi AJ, Treichel H, Cansian RL, Ghisleni CP, et al. Avaliação das condições microbiológicas de uma unidade de alimentação e nutrição. Alim Nutr. 2009; 20(4):663-8.

17. Pragle AS, Harding AK, Mack JC. Workers' perspective on handwashing behaviors and barriers in the restaurant environment. Environ Health. 2007; 69(10):27-32.

18. Teo CRPA, Corrêa EN, Gallina LS, Fransozi C. Programa nacional de alimentação escolar: adesão, aceitação e condições de distribuição de alimentação na escola. Nutrire. 2009; 34(3):165-85.

19. Brasil. Conselho Nacional de Educação [Internet]. Resolução CNE/CES n 5, de 7 de novembro de 2001. [acesso 2010 dez 15]. Disponível em: <http:// www.asbran.org.br/sitenovo/arquivos/resolucao CNECES5.pdf>.

20. Esperança LC, Marchioni DML. Qualidade na produção de refeições em restaurantes comerciais na região de Cerqueira César, São Paulo. Nutrire. 2011; 36(1):71-83.
21. Ebone MV, Cavalli SB, Lopes SJ. Segurança e qualidade higiênico-sanitária em unidades produtoras de refeições comerciais. Rev Nutr. 2011; 24(5): 725-34. doi: 10.1590/S1415-527320110005000 06.

22. Cardoso RCV, Souza EVA, Santos PQ. Unidades de alimentação e nutrição nos campi da Universidade Federal da Bahia: um estudo sob a perspectiva do alimento seguro. Rev Nutr. 2005; 18(5):669-80. doi: 10.1590/S1415-52732005000500010.

23. Figueiredo RM. As armadilhas de uma cozinha. $3^{a}$ ed. São Paulo: Manole; 2002.

24. Leite CL, Cardoso RCV, Góes JAW, Figueiredo KVNA, Silva EO, Bezerril MM, et al. Formação para merendeiras: uma proposta metodológica aplicada em escolas estaduais atendidas pelo programa nacional de alimentação escolar, em Salvador, Bahia. Rev Nutr. 2011; 24(2):275-85. doi: 10.1590/\$1415-5 2732011000200008

25. Kraemer FB, Aguiar OB. Gestão de competências e qualificação profissional no segmento da alimentação coletiva. Rev Nutr. 2009; 22(5):609-19. doi: 10.1590/S1415-52732009000500002.

26. Gonzalez CD, Perrella NG, Rodrigues RL, Gollücke APB, Schattan RB, Toledo LP. Conhecimento e percepção de risco sobre higiene alimentar em manipuladores de alimentos de restaurantes comerciais. Nutrire. 2009; 34(3):45-56.

27. Clayton DA, Griffith CJ, Price P, Peters AC. Food handlers' beliefs and self-reported practices. Int J Environ Health Res. 2002; 12(1):25-39. doi:10.10 80/09603120120110031.

28. Park S-H, Kwak T-K, Chang H-J. Evaluation of the food safety training for food handlers in restaurant operations. Nutr Res Pract. 2010; 4(1):58-68.

29. Medeiros CO, Cavalli SB, Salay E, Proença RPC. Assessment of the methodological strategies adopted by food safety training programmes for food service workers: a systematic review. Food Control. 2011; 22:1136-44. doi:10.1016/j.foodcont. 2011.02.008.

30. Ba ş M, Ersun AS, Kivanç G. The evaluation of food hygiene knowledge, attitudes, and practices of food handlers' in food business in Turkey. Food Control. 2006; 17:317-22. doi:10.1016/j.foodcont. 2004.11.00.

31. Santos LAS, Paiva JB, Mello AL, Fontes GAV, Sampaio LR, Freitas MCS. O nutricionista no programa de alimentação escolar: avação de uma experiência de formação a partir de grupos focais. Rev Nutr. 2012; 25(1):107-17. doi: 10.1590/S1415-5273012 000100010. 
